\title{
Vins et vignerons biologiques en France, une approche territoriale
}

Les exemples du Bordelais et de la Vallée du Rhône

\section{Frédérique Célérier}

\section{(2) OpenEdition}

Journals

\section{Édition électronique}

URL : http://journals.openedition.org/cdg/1186

DOI : $10.4000 /$ cdg. 1186

ISSN : 2107-7266

Éditeur

UMR 245 - CESSMA

\section{Référence électronique}

Frédérique Célérier, «Vins et vignerons biologiques en France, une approche territoriale », Carnets de géographes [En ligne], 10 | 2017, mis en ligne le 30 septembre 2017, consulté le 24 septembre 2020. URL : http://journals.openedition.org/cdg/1186 ; DOI : https://doi.org/10.4000/cdg.1186

Ce document a été généré automatiquement le 24 septembre 2020.

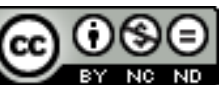

La revue Carnets de géographes est mise à disposition selon les termes de la Licence Creative Commons Attribution - Pas d'Utilisation Commerciale - Pas de Modification 4.0 International. 


\title{
Vins et vignerons biologiques en France, une approche territoriale
}

\author{
Les exemples du Bordelais et de la Vallée du Rhône
}

Frédérique Célérier

1 Ma thèse met au jour les logiques spatiales et temporelles à l'œuvre dans la diffusion de la viticulture biologique en France, lame de fond dont les mutations vont au-delà de la seule finalité environnementale. La viticulture biologique répond à des déterminants pluriels, économiques, gustatifs, éthiques, sanitaires, qui manifestent un renouvellement global du lien entre vin, viticulture et territoire.

2 Née au lendemain de la Seconde Guerre mondiale et longtemps restée marginale, la viticulture biologique connaît depuis une dizaine d'années un essor remarquable en France. L'engouement pour les vins biologiques, tant dans leur production que dans leur consommation, est porté par la progression des préoccupations environnementales vis-à-vis de l'utilisation de pesticides, et par une critique croissante de la standardisation des modes de fabrication du vin. Pourtant, au sein du monde vitivinicole, la viticulture biologique peine à s'imposer comme une réponse pertinente à ces enjeux d'actualité. Sa progression en demi-teinte questionne la capacité des acteurs des territoires vitivinicoles à s'en saisir.

3 Ma problématique a ainsi été d'envisager dans quelle mesure les modalités de l'émergence et de la diffusion de la viticulture biologique en France, de la portée et des limites de cette diffusion, sont le témoin et le moteur de transformations des relations entre villes et campagnes. L'objectif principal de ma recherche était donc de comprendre la façon dont le monde vitivinicole se saisit des attentes de la société à l'égard du vin, des vignobles et des vignerons qui les produisent.

4 Mon travail s'inscrit dans les questionnements d'une géographie rurale française en profond renouvellement, autour des processus de construction territoriale qui se mettent en place autour des produits agricoles dits tantôt de qualité, tantôt de terroir, ancrés dans des territoires de production délimités. Considérant que la démarche biologique s'inscrit dans cette dynamique de reterritorialisation de l'agriculture, j'ai été amenée à réfléchir aux aménités et aux services potentiels et réels offerts par la 
viticulture biologique dans ses territoires, en m'appuyant notamment sur le corpus théorique de la ressource territoriale et sur l'analyse des nouvelles fonctions des espaces agricoles dans une société urbanisée. J'ai ensuite voulu montrer que cette diffusion de la viticulture biologique s'inscrivait dans la "nouvelle planète des vins " qui se fait jour depuis la fin du XXe siècle et dans l'exacerbation des concurrences entre anciens et nouveaux pays producteurs. Je me suis appuyée sur un corpus de géographie vitivinicole qui analyse ces bouleversement récents, sans négliger l'exploration de la littérature d'histoire vitivinicole, afin de resituer la diffusion de la bio dans le temps long de la construction des territoires du vin. Enfin, j'ai complété mon approche par des éclairages venus d'autres disciplines afin de mieux comprendre certains déterminants de diffusion de la viticulture bio. La mobilisation des travaux de sociologie a été déterminante, dans la compréhension des enjeux de santé, du «coût humain" des pesticides, ainsi que dans l'analyse des dynamiques collectives se structurant autour de la défense d'une autre agriculture, et dans le rôle ambivalent tenu par les consommateurs dans ces mouvements.

5 Afin de répondre à ces objectifs, j'ai choisi de faire appel à une pluralité de sources, dont je me suis emparée à travers une méthodologie avant tout qualitative. J'ai essentiellement travaillé à partir des 105 entretiens menés auprès des vignerons et d'autres acteurs du vignoble (des Chambres d'agriculture aux collectivités territoriales en passant par les interprofessions de la vigne et du vin et les organismes de développement de la bio). Ce matériau empirique a été croisé à la littérature scientifique évoquée, ainsi qu'à une multitude de sources de diverse nature (littérature technique, publicitaire, administrative, médiatique), une documentation foisonnante permettant d'embrasser cette totalité qu'est la viticulture biologique. Toutes ces données m'ont permis de réaliser une cartographie originale, à différentes échelles.

6 Mon travail repose sur la constitution d'un corpus unique de données empiriques à partir de deux terrains, les vignobles bordelais et rhodanien, et ce afin d'analyser un processus à l'œuvre dans l'ensemble du vignoble français. Il ne s'agit pas d'une comparaison au sens strict. J'ai souhaité expliquer un même phénomène à l'aune de plusieurs situations. Ce choix procède aussi de l'idée qu'aucune partie du vignoble français ne peut s'envisager aujourd'hui de manière isolée. La mondialisation de l'économie viticole met en concurrence entre elles les régions viticoles qui composent le vignoble français. Leur capacité à se saisir des questions environnementales participe de cette rivalité. Les dynamiques de réseaux, les mobilités au sein des vignobles, invitent à sortir d'un terrain unique.

Le choix des terrains d'étude s'est effectué parmi les régions viticoles qui connaissent un essor important, rapide mais non linéaire de leur production biologique. Les deux vignobles ont connu des trajectoires fort différentes. Le Bordelais, l'un des principaux vignobles biologiques de France, est situé dans le plus grand département viticole. Le développement de la viticulture biologique y apparaît pourtant comme hésitant. Le vignoble de la Vallée du Rhône est beaucoup plus engagé dans la viticulture biologique, mais aussi de moindre réputation que le Bordelais. Ce renversement de hiérarchie étonnant masque des réalités locales complexes. Ainsi ai-je tenté de me départir de l'analyse régionale d'une part, et de me décentrer des vignobles les plus prestigieux d'autre part. Ce choix s'est aussi fondé sur le souci d'explorer le plus de situations possibles. 
8 Au sein de ces deux terrains, j'ai fait le choix de faire des vignerons les briques de base de mon approche, et par conséquent de mon travail d'enquête. Si les recompositions induites par la viticulture biologique engagent l'ensemble des acteurs des vignobles, mon hypothèse était que les vignerons sont les principaux initiateurs de ces transformations. Sans négliger le rôle des pouvoirs publics ou encore des consommateurs, ce postulat me permettait de ne pas cantonner les agriculteurs dans un rôle passif face à ces attentes sociétales, ou de réduire la diffusion de la bio à une adaptation des campagnes aux injonctions urbaines. Ce choix me permettait ainsi de m'inscrire dans les approches humaines, voire humanistes, de la vigne et du vin, qui valorisent l'ingéniosité des vignerons, non seulement face aux contraintes du milieu, mais aussi face aux aspirations de la société. Dans cette perspective, la bio donne un nouveau sens à l'idée d'une viticulture "objet du bon vouloir humain", selon la formule de Roger Dion. C'est ce caractère initiateur, et le rôle du travail humain, que je voulais réhabiliter en faisant ce choix. La richesse des données issues des entretiens menés auprès des vignerons bio et en biodynamie rend compte de la pertinence d'interroger les pratiques, les discours et les représentations dans l'analyse de ce phénomène, et de la diffusion d'une innovation, plus largement.

\section{Discipline \\ Géographie}

\section{Directeur}

Mayté Banzo, Professeur des Universités, Université Bordeaux Montaigne, directrice

Raphaël Schirmer, Maître de conférences, Université Bordeaux Montaigne, codirecteur

\section{Université}

Université Bordeaux Montaigne

Membres du jury de thèse, soutenue le 8 décembre 2016

Mayté Banzo, Professeur des Universités, Université Bordeaux Montaigne, directrice

Estelle Deléage, Maître de conférences, Université de Caen Normandie Claire Delfosse, Professeur des Universités, Université Lyon 2

Michaël Pouzenc, Professeur des Universités, Université Toulouse - Jean Jaurès, rapporteur

Laurent Rieutort, Professeur des Universités, Université Blaise-Pascal, ClermontFerrand, rapporteur

Raphaël Schirmer, Maître de conférences, Université Bordeaux Montaigne, codirecteur

Situation professionnelle à l'issue de la thèse :

Attachée Temporaire d'Enseignement et de Recherche à l'Université d'Évry-Vald'Essonne

\section{Courriel}

frederique.celerier[at]gmail.com 
INDEX

Thèmes : Carnets de soutenances 